\title{
AC losses in Sn-doped Bil.6Pb0.4Sr2(Ca1-xSnx)2Cu3Od superconductors
}

\begin{abstract}
Measurements of complex AC susceptibility $G=G+G$ as a function of temperature have been carried out on $\mathrm{Sn}$-doped Bi1.6Pb0.4Sr2(Ca1-xSnx)2Cu3Oŭ superconductor samples prepared via the conventional solid state reaction method. All the samples exhibit perfect diamagnetism below $109 \mathrm{~K}$. The G (T) curves display two-step features, indicating the presence of mixed phases and therefore weakening of the grains' coupling. The amount of shielded volume in $\mathrm{Sn}$-free samples is greater than that in Sn-doped samples. The intrinsic peak due to the small AC losses within the grain was not observed in the $\mathrm{G}(\mathrm{T})$ curves for all samples. However, the coupling peak, TP, for Sn-free samples at an applied field of $1.0 \mathrm{Oe}$ was observed at $89 \mathrm{~K}$ and shifted to a lower temperature ranging from $59 \mathrm{~K}$ to $64.2 \mathrm{~K}$ in Sndoped samples. The amounts of hysteresis losses above the TP in all doped samples were smaller than that of the Sn-free sample. Therefore, the effect of Sn doping suppressed the inter-granular critical current, Jcm, and the presence of weak links that coupled the superconducting grains.
\end{abstract}

Keyword: AC losses; BSCCO; Sn-doped; Superconductor 